\title{
Modelagem do teor de carbono orgânico em solos de fragmentos de cerrado de Januária e Bonito de Minas, Minas Gerais
}

Vinícius Augusto Morais ${ }^{1}$, Carlos Alberto Silva ${ }^{1}$, José Roberto Soares Scolforo ${ }^{1}$, José Márcio de Mello ${ }^{1}$, Emanuel José Gomes de Araújo $^{2}$, Edson Aquiles de Assis ${ }^{1}$

${ }^{1}$ Universidade Federal de Lavras, CP 3037, CEP 37200-000, Lavras, MG, Brasil

${ }^{2}$ Universidade Federal Rural do Rio de Janeiro, Departamento de Silvicultura, Instituto de Florestas, BR 465, km 07, CEP 23890-000, Seropédica, RJ, Brasil

"Autor correspondente:
vemorais@bol.com.br

Termos para indexação:

Modelagem

Mudanças climáticas

Raiz serrapilheira

Carbono orgânico total

\section{Index terms: \\ Modeling \\ Climatic change \\ Root \\ Litter \\ Total organic carbon}

Histórico do artigo:

Recebido em 02/04/2013

Aprovado em 10/12/2013

Publicado em 31/12/2013

doi: $10.4336 / 2013 . p f b .33 .76 .507$
Resumo - O carbono orgânico total do solo (C) é regulado por fatores climáticos, altitude, textura e profundidade do solo. Em função do maior armazenamento em superfície, é comum o modelo exponencial se ajustar aos teores de $\mathrm{C}$ do perfil de solo. Objetivou-se estudar a influência de fatores climáticos, altitude e da profundidade sobre o $\mathrm{C}$ armazenado em solos de cinco fragmentos de cerrado localizados nos Municípios de Januária e Bonito de Minas, MG. Amostras de solo foram coletadas nas camadas de 0-10, 10-20, 20-40, 40-60 e 60-100 cm de profundidade. Os teores máximo e mínimo de $\mathrm{C}$ variam em função do local de coleta, fragmento e camada de solo amostrada; maiores variações nos teores de $C$ são notadas para a camada de $0-10 \mathrm{~cm}$, cujos teores de $\mathrm{C}$ não se correlacionam com a biomassa de raiz e de serrapilheira e com outras variáveis condicionantes do C-solo testadas. São disponibilizadas equações logarítmicas específicas para cada um dos cinco fragmentos, para se estimar os teores de carbono a partir da temperatura, precipitação, altitude e camada de solo, até a profundidade de $100 \mathrm{~cm}$.

\section{Modeling of the organic carbon contents in soils of cerrado} fragments located in Januária and Bonito de Minas, Minas Gerais

\footnotetext{
Abstract - The total soil organic carbon (C) is regulated by climatic factors, altitude, texture and soil depth. Due to higher storage in surface, it is common exponential model fit to the $\mathrm{C}$ content in the soil profile. This study was carried out in order to study the influence of climatic factors, altitude and soil depth on the vertical distribution (0-100 $\mathrm{cm}$ ) of $\mathrm{C}$ in five cerrado fragments located in the municipalities of Januária and Bonito de Minas, MG. Soil samples were collected in the following layers: 0-10, 10-20, 20-40, 40-60 and 60-100 $\mathrm{cm}$. The maximum and minimum levels of $\mathrm{C}$ varies depending on the sampling site, fragment and soil layer sampled; larger variations in the levels of $\mathrm{C}$ are noted for the $0-10 \mathrm{~cm}$ layer, whose $\mathrm{C}$ content did not correlate with root and litter biomasses, and with other soil $\mathrm{C}$ conditioning factors tested. Specific logarithmic equations are provided for each of the five fragments in order to estimate the $\mathrm{C}$ contents (in the $0-100 \mathrm{~cm}$ soil layers) as a function of the average temperature, precipitation, altitude and fragment soil layer sampled.
} 


\section{Introdução}

As mudanças do uso da terra e o desmatamento são práticas que contribuem para o fluxo de carbono do solo para a atmosfera e que, em Minas Gerais, respondem por $54 \%$ das emissões de gases de efeito estufa (Fundação de Ciência, Aplicações e Tecnologias Espaciais, 2010). No Estado, são escassos os estudos que investigam os padrões de retenção de $\mathrm{C}$ nos perfis de solo, tendo em vista o papel central desempenhado pela matéria orgânica em regular a qualidade do solo e os ciclos biogeoquímicos de diversos elementos químicos (Stevenson, 1994; Dick \& Gregorich, 2004). Além disso, o C armazenado em solos tropicais representa $32 \%$ do C retido nos solos da Terra (Lal, 2002) e o estudo da fitofisionomia Cerrado é importante, uma vez que este bioma engloba cerca de $25 \%$ da área do território brasileiro e $28 \%$ da cobertura vegetal de Minas Gerais (Scolforo \& Carvalho, 2008) e, rapidamente, vem sendo transformado em novas fronteiras agrícolas para produção de alimentos.

A determinação dos teores de $\mathrm{C}$ no solo é dispendiosa e requer tempo e recursos financeiros, por empregar técnicas de coleta que são desgastantes aos responsáveis pelas coletas, quando realizadas em profundidades abaixo dos $40 \mathrm{~cm}$. Assim, torna-se necessário o desenvolvimento de técnicas alternativas a essas coletas, e uma dessas alternativas é a modelagem de teores de C em perfis de solos representativos do bioma Cerrado de Minas Gerais. Em função do desenvolvimento de modelo matemático, a variável dependente (teores de carbono), em geral, de difícil coleta, é estimada a partir de variáveis independentes, ou seja, a partir das condicionantes dos teores de $\mathrm{C}$ em solo. Com o uso de funções matemáticas a concentração de $\mathrm{C}$ pode ser estimada a partir de variáveis climáticas, da profundidade do solo, textura, densidade e vários outros atributos do solo (Arrouays \& Pelissier, 1994; Kay et al., 1997; Tomasella \& Hodnett, 2004; Zinn et al., 2005; McBratney et al., 2010). Estas funções já foram utilizadas para estimativa da densidade do solo (Kaur et al., 2002; Benites et al., 2007; Martin et al., 2009; Marcolin \& Klein, 2011) e, em menor escala, do carbono orgânico no solo (Arrouays \& Pelissier, 1994; Kay et al., 1997; Zinn et al., 2005).

Em sistemas naturais, os níveis de $\mathrm{C}$ presentes nos solos são condicionados pela textura, vegetação (aporte de biomassa), temperatura média do ar, precipitação, altitude e relevo (Stevenson, 1994; Quideau, 2002).
De fato, a concentração de carbono no solo é função de uma variedade de fatores, mas, no entanto, o clima apresenta um efeito mais pronunciado (Lal, 2005). A precipitação apresenta relação positiva com o teor de carbono no solo, em função da maior disponibilidade de água implicar em maior produção de biomassa, o que significa maior aporte de $\mathrm{C}$ ao solo (Stevenson, 1994). O aumento da temperatura implica em menor armazenamento de $\mathrm{C}$ no solo, em razão da elevação da taxa de decomposição da matéria orgânica em locais mais quentes (Corazza et al., 1999; Jobbágy \& Jackson, 2000; Lal, 2005; Salton et al., 2011). O aumento de altitude implica em maior armazenamento de $\mathrm{C}$ em solo, em razão da queda da temperatura média em regiões mais altas e, por conseguinte, da menor decomposição da matéria orgânica devido a menor atividade biológica (Stevenson, 1994).

O C poder ser também modelado em função da profundidade do solo. Diversos autores investigaram a distribuição do carbono no perfil de solo e concluíram que os teores de $\mathrm{C}$ variam de uma camada de solo para outra (Roscoe \& Machado 2002; Aduan et al., 2003; Lal, 2005; Paiva \& Faria, 2007; Paiva et al., 2011), de modo que há maior concentração de matéria orgânica nas camadas superficiais, em relação ao subsolo, sendo o modelo exponencial o que melhor explica a distribuição do C no perfil de solo (Arrouays \& Pelissier, 1994; Richter et al., 1999; Jobbágy \& Jackson, 2000; Zhou et al., 2006; Bayer et al., 2006; Salton et al., 2011). Esses padrões de distribuição de $\mathrm{C}$ variam de um local para outro, sendo observado, em geral, maior concentração de $\mathrm{C}$ em superfície e queda drástica dos teores de $\mathrm{C}$ com o aumento da profundidade do solo. Não se trata, contudo, de padrão estabelecido, tendo em vista que, em alguns locais, o C é maior nas camadas superiores de solo, mas não varia muito à medida que se aprofunda no perfil de solo. Essas variações na distribuição vertical do C-solo assumem importância e merecem ser estudadas, uma vez que, num cenário eventual de aquecimento global e de aumento da temperatura média do planeta, o C armazenado em camada mais profundas pode permanece por mais tempo sequestrado no solo (Knops \& Bradley, 2009).

Objetivou-se a modelagem do teor de carbono em função da profundidade do solo e de dados climáticos e de altitude, em solos de fragmentos de cerrado sensu stricto dos Municípios de Januária e Bonito de Minas, MG. 


\section{Material e métodos}

\section{Caracterização das áreas de estudo}

Os fragmentos florestais amostrados para esse estudo fazem parte da rede de parcelas permanentes do Inventário Florestal de Minas Gerais (Scolforo et al., 2008) e estão localizados na região norte do Estado de Minas Gerais, Brasil, nos Municípios de Januária e Bonito de Minas (Tabela 1 e Figura 1). A fitofisionomia predominante nos fragmentos é o cerrado sensu stricto, o qual se caracteriza por vegetação composta, predominantemente, de um estrato arbóreo-arbustivo e outro, em menor ocorrência, herbáceo-subarbustivo, ambos bem desenvolvidos (Marimon Júnior \& Haridasan, 2005; Ribeiro \& Walter, 2008).

Tabela 1. Caracterização das áreas de estudo e número de parcelas amostradas por fragmento de cerrado.

\begin{tabular}{lccccc}
\hline \multirow{2}{*}{ Característica } & \multicolumn{5}{c}{ Fragmento } \\
\cline { 2 - 6 } & $\mathbf{1}$ & $\mathbf{2}$ & $\mathbf{3}$ & $\mathbf{4}$ & $\mathbf{5}$ \\
\hline Município & Bonito de Minas & Januária & Januária & Januária & Januária \\
Latitude (S) & $15^{\circ} 20^{\prime}$ & $15^{\circ} 49^{\prime}$ & $15^{\circ} 50^{\prime}$ & $15^{\circ} 20^{\prime}$ & $14^{\circ} 59^{\prime}$ \\
Longitude (W) & $44^{\circ} 58^{\prime}$ & $44^{\circ} 35^{\prime}$ & $44^{\circ} 36^{\prime}$ & $45^{\circ} 14^{\prime}$ & $45^{\circ} 24^{\prime}$ \\
Área (ha) & 253 & 56,7 & 74,9 & 487 & 336 \\
Altitude média (m) & 588 & 512 & 508 & 620 & 758 \\
Precipitação média $(\mathrm{mm})$ & 1073 & 1076 & 1076 & 1098 & 1101 \\
Temperatura média $\left({ }^{\circ} \mathrm{C}\right)$ & 23,6 & 24,2 & 24,2 & 23,4 & 22,6 \\
Parcelas amostradas por fragmentos & 6 & 5 & 4 & 10 & 7 \\
\hline
\end{tabular}

Fonte: Scolforo et al. (2008).

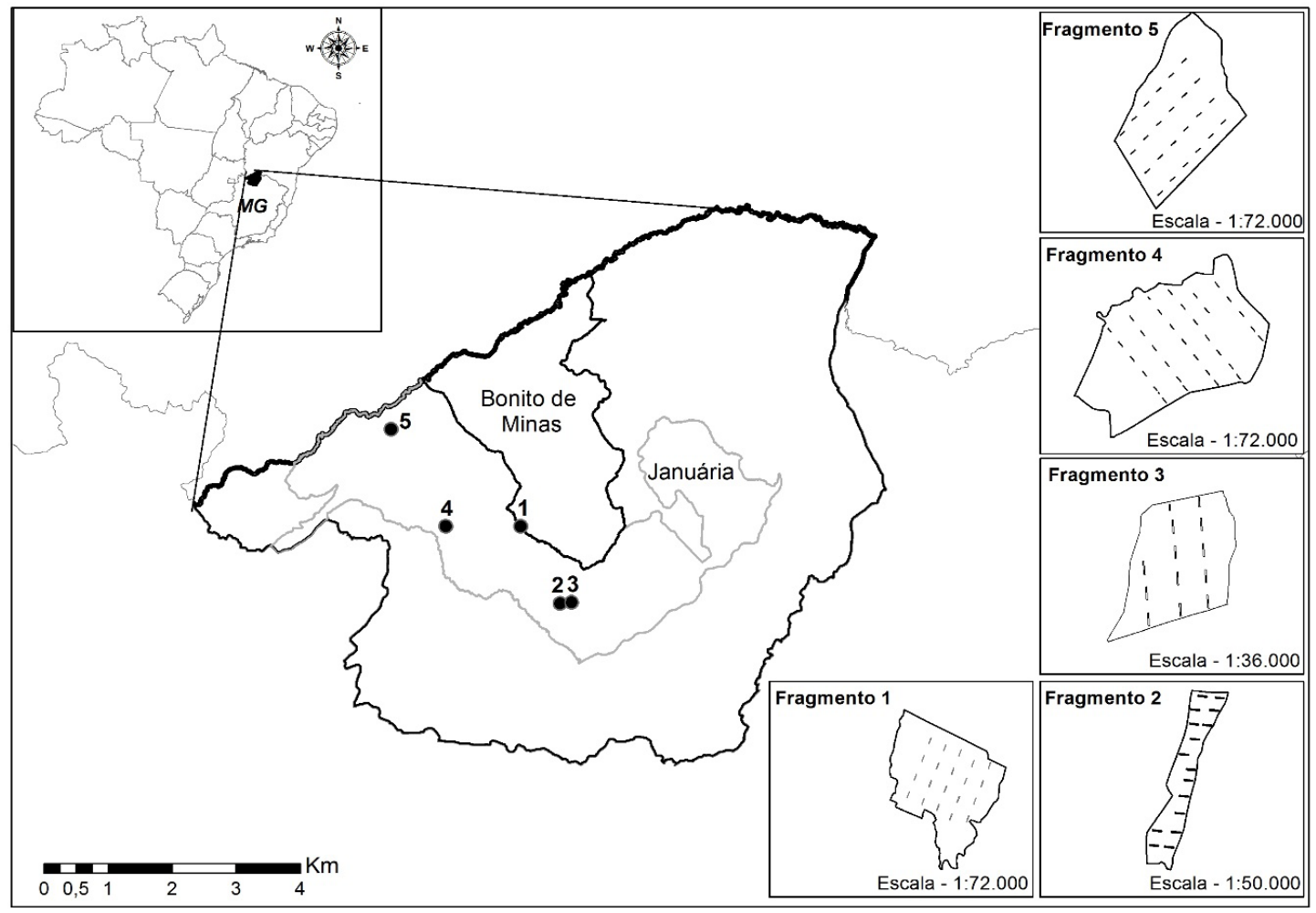

Figura 1. Mapa de localização dos cinco fragmentos de cerrado avaliados nos Municípios de Januária e Bonito de Minas, MG, e contorno de cada fragmento estudado, com localização de suas respectivas parcelas de amostragem do solo. 
O clima predominante na região dos fragmentos, conforme a classificação climática de Thornthwaite, é o C1- subúmido seco, o qual se caracteriza por índices de umidade (Iu) no intervalo de $-33,3 \leq \mathrm{Iu}<0$ (Carvalho et al., 2008). O tipo de solo predominante é o Latossolo Vermelho-Amarelo, que pode ser descrito como um solo profundo bastante intemperizado, com baixa fertilidade natural (Curi et al., 2008) e, geralmente, com predomínio na fração argila de caulinita e oxi-hidróxidos de ferro e de alumínio, sendo a textura do solo do fragmento 1 caracterizada como argilosa e a dos demais fragmentos, como média. Demais características do solo são apresentadas na Tabela 2.

\section{Coleta e preparo das amostras}

Foram definidas 32 unidades amostrais (parcelas), distribuídas aleatoriamente nos cinco fragmentos de cerrado. Em cada ponto amostral, alocou-se um gabarito de aço, com área de $0,5 \mathrm{~m}^{2}(0,5 \times 1 \mathrm{~m})$, onde foram coletadas as amostras de serrapilheira, raízes e solo. Para os materiais vegetais, foi determinada a biomassa, visando a utilização desses dados como possíveis fatores condicionantes do $\mathrm{C}$ armazenado em solo e, para o solo, foi determinado o teor de $\mathrm{C}$.

Determinado o ponto para coleta do material (Morais et al., 2013), alocou-se um gabarito de aço e toda a serapilheira, presente na área do mesmo, foi coletada.

Tabela 2. Atributos de fertilidade, textura e densidade (DS) das amostras coletadas em cinco profundidades dos solos pertencentes aos cinco fragmentos de cerrado sensu stricto amostrados.

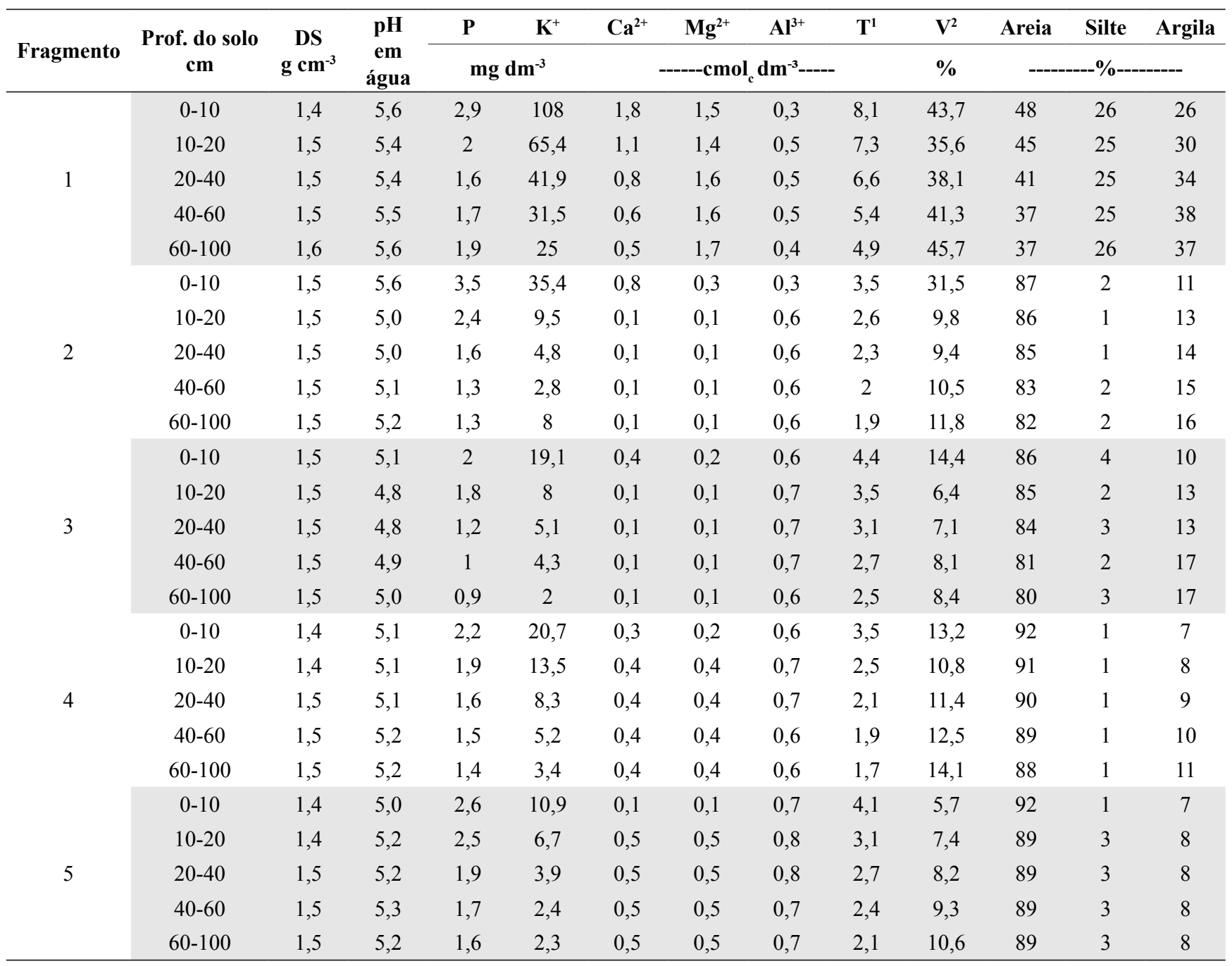

${ }^{1} \mathrm{~T}=$ capacidade de troca de cátions a $\mathrm{pH} 7 ;{ }^{2} \mathrm{~V}=$ saturação por bases. 
Posteriormente, procedeu-se a abertura de uma trincheira e todas as raízes presentes na área do gabarito $(0,5 \times 1$ $\mathrm{m})$ e no perfil de solo $(0-100 \mathrm{~cm})$ para cinco camadas de solo: 0-10, 10-20, 20-40, 40-60 e 60-100 cm foram coletadas. Em cada camada, as raízes foram separadas conforme a espessura: $<5 \mathrm{~mm}$; 5,1 a $10 \mathrm{~mm}$; e $>$ que $10 \mathrm{~mm}$. As amostras de solo, para a análise do teor de carbono, determinação da densidade e caracterizações física e química (Tabela 2), foram coletadas nas mesmas camadas mencionadas para as raízes.

No laboratório, as amostras coletadas de serrapilheira e raízes foram lavadas e secas em estufa com circulação forçada de ar, à temperatura de $65-70^{\circ} \mathrm{C}$, até atingirem peso constante. A biomassa seca ( $\mathrm{g}$ ) de cada amostra foi quantificada, utilizando-se balança de $0,01 \mathrm{~g}$ de precisão. Para análise do teor de carbono, as amostras de solo foram maceradas em gral de porcelana, com o uso de pistilo, secas ao ar e peneiradas (peneira de diâmetro de malha de $0,250 \mathrm{~mm}$ ). A densidade foi determinada relacionando o volume do solo coletado com seu respectivo peso seco; as determinações de atributos químicos do solo foram realizadas no Laboratório de Fertilidade da Universidade Federal de Lavras.

O carbono foi analisado por combustão seca, em analisador do tipo TOC (total organic carbon), modelo Vario Cube, marca Elementar. Na análise por combustão a seco, foram utilizadas de 10 a $12 \mathrm{mg}$ de amostra de solo. As amostras foram acondicionadas em cápsulas de estanho e incineradas em forno a $950^{\circ} \mathrm{C}$. $\mathrm{O}$ gás $\mathrm{CO}_{2}$ gerado por cada amostra foi quantificado por um detector de infravermelho do tipo NDIR, de modo que o teor de $\mathrm{C}$ foi calculado relacionando-se o $\mathrm{CO}_{2}$ evoluído com a massa de amostra analisada.

\section{Análise exploratória dos dados e modelagem}

Os dados foram submetidos a uma análise preliminar e, posteriormente, as principais características edafoclimáticas (temperatura média do ar e precipitação), altitude do ponto amostral e as biomassas de serrapilheira e de raízes foram correlacionadas com os teores de carbono determinados para as diferentes camadas de solo. Essa etapa foi realizada com vistas à seleção das principais variáveis condicionantes da distribuição dos teores de $\mathrm{C}$ no perfil de solo.

Como os teores de carbono no solo variam em razão de sua distribuição no perfil, os mesmos foram sujeitos à modelagem, considerando-se os teores de $\mathrm{C}$ nas diferentes camadas amostradas em cada fragmento. Inicialmente, os dados foram plotados em gráficos de dispersão. Em seguida, foram ajustados modelos de regressão não linear (logarítmico (1)) para cada fragmento, ou seja, cinco modelos foram disponibilizados, a fim de que a distribuição vertical do $\mathrm{C}$ em função da profundidade do solo fosse matematicamente descrita.

Onde: $y=$ variável dependente, $\mathrm{C}(\%) ; a=$ constante

$$
y=a+b * \ln (x)
$$

de regressão; $b=$ coeficiente de regressão; $\ln =$ logaritmo natural; $x=$ variável independente, profundidade do solo $(\mathrm{cm})$.

Também foram calculadas as médias aritmética e ponderada dos teores de $\mathrm{C}$ para as camadas de $10 \mathrm{~cm}$ até $100 \mathrm{~cm}$ de profundidade e, então, foi calculada a razão entre o teor médio de $\mathrm{C}$ na camada de solo de $0-10 \mathrm{~cm}$ com o $\mathrm{C}$ médio da camada $0-100 \mathrm{~cm}$. Com esse procedimento foi calculado o fator de concentração do $\mathrm{C}$ em superfície. Posteriormente, foram plotadas em um gráfico as médias aritmética e ponderada, e os valores máximo e mínimo para o teor de $\mathrm{C}$ de cada fragmento.

A fim de se estudar a influência de fatores condicionantes da matéria orgânica no ambiente sobre os teores de $\mathrm{C}$ determinados para diferentes camadas de solo dos cinco fragmentos investigados, foram ajustados modelos de regressão linear múltiplos pelo método dos mínimos quadrados ordinários, utilizandose, para isso, o software $R$ (R Development Core Team, 2011). Esses modelos relacionaram os teores de $\mathrm{C}$ com precipitação e temperatura, altitude do ponto de coleta $\mathrm{e}$ com características físicas e químicas dos solos, além da biomassa das raízes e de serapilheira, sendo escolhidas, para modelagem, as variáveis que, em função do grau de significância da correlação e do maior coeficiente de correlação, mais explicam as variações nos teores de $\mathrm{C}$ em diferentes camadas de solo. A precisão dos ajustes foi analisada considerando-se o coeficiente de determinação ajustado $\left(\mathrm{R}_{\text {ajust. }}^{2}\right.$ ), média dos resíduos (ME), desvio padrão do erro da estimativa (DPEEs), raiz do erro médio da estimativa (RME) e o gráfico de resíduos. As fórmulas para cálculo dos parâmetros estatísticos mencionados são apresentadas a seguir (Charnet et al., 2008).

$$
\begin{aligned}
& R^{2}=\frac{\left[\operatorname{cov}\left(y_{i}, \hat{y}_{i}\right)\right]^{2}}{\operatorname{var}\left(y_{i}\right) \operatorname{var}\left(\hat{y}_{i}\right)} \\
& M E=\frac{1}{n} \sum_{i=1}^{n}\left(\hat{y}_{i-} y_{i}\right)
\end{aligned}
$$




$$
\begin{aligned}
& \text { DPEEs }=\sqrt{\frac{1}{n-1} \sum_{i=1}^{n}\left[\left(\hat{y}_{i-} y_{i}\right)-M E\right]^{2}} \\
& R M E=\sqrt{\frac{1}{n} \sum_{i=1}^{n}\left(\hat{y}_{i-} y_{i}\right)^{2}}
\end{aligned}
$$

onde: $y_{i}$ e $\hat{y}_{i}$ são os teores de carbono observados e estimados para cada observação, respectivamente.

\section{Resultados e discussão}

Analisando-se todas as amostras, independentemente da profundidade de coleta, os teores de carbono no solo variaram de 0,15 a 1,38\%, com média de $0,51 \pm 0,24 \%$ (Tabela 3). Os valores observados são relativamente baixos e apresentam grande amplitude de variação, mas refletem os teores listados por Zinn et al. (2005). No entanto, são menores que os teores apresentados por Oliveira et al. (2001) e Freixo et al. (2002), para solos de cerrado brasileiro.

Nas camadas de solo de 0-10 e 10-20 cm, foram verificados os maiores teores de $\mathrm{C}$ nos perfis avaliados e também a maior variabilidade de teores de C. A amplitude de variação dos teores de $\mathrm{C}$ diminuiu a partir desta profundidade, com exceção do fragmento 5, que apresentou menor variação quanto à distribuição vertical do C. No fragmento 5, os teores de C em superfície e na última camada de solo diferem, mas a queda de teores não é tão brusca quanto a notada para solos de outros fragmentos. Em geral, há uma queda nos teores de C à medida que se aprofunda nos perfis de solos, cuja amplitude de variação é peculiar para cada fragmento, não é linear e apresenta distribuição que se ajusta mais a modelos matemáticos logarítmicos e exponenciais.

Na Tabela 4 são apresentados os dados de biomassa de serrapilheira e de raízes, essa última, por camada de solo. A biomassa de serrapilheira variou de 3,42 $\mathrm{t} \mathrm{ha}^{-1}$ (fragmento 2) a 9,36 t ha-1 (fragmento 4). A biomassa de raiz varia em função da camada de solo e do fragmento amostrados, com maior massa de raízes nas camadas superficiais de solo, até $20 \mathrm{~cm}$ de profundidade. Para as raízes, há uma queda exponencial da biomassa à medida que se aprofunda no perfil de solo. Cumulativamente, o fragmento 1 é o que possui menor biomassa de raízes na camada de solo de $0-100 \mathrm{~cm}$. No fragmento 5, é notada a maior biomassa acumulada de raízes.

Mesmo com grande variação nos estoques de de biomassa de raízes e serapilheira, foi verificada correlação positiva significativa desses índices com os teores de carbono nas camadas de 0-10 e 10-20 $\mathrm{cm}$, portanto, a biomassa de raízes e de serrapilheira explicam cerca de $50 \%$ das variações nos teores de C na superfície do solo (Tabela 5). Segundo Lal (2005), Leifeld et al. (2005) e Sheikh et al. (2009), os teores de carbono no solo são função da complexa interação entre clima, solos e espécies arbóreas, dependendo também do aporte de $\mathrm{C}$ e de outros nutrientes pela serrapilheira. Jobbágy \& Jackson (2000) salientam que as espécies arbóreas exercem grande influência nos estoques de matéria orgânica do solo, em função das taxas de decomposição e aporte de restos vegetais ao solo, além de regularem a distribuição vertical de raízes $\mathrm{e}$, por conseguinte, o aporte de $\mathrm{C}$ e de seus compostos no perfil do solo. Segundo Corazza et al. (1999), Lal (2005, 2008) e Salton et al. (2011), a diferença entre acúmulo de resíduos sobre o solo e a quantidade de raízes finas encontradas na camada superficial explica, em parte, a grande variação observada nos teores de $\mathrm{C}$ em solo.

Quando analisado cada ponto de coleta, foi possível verificar que o teor de $\mathrm{C}$ no perfil do solo ajusta-se ao modelo matemático do tipo logarítmico, o qual materializa o decréscimo nos teores de $\mathrm{C}$ à medida que se aprofunda no perfil de solo. Essa distribuição vertical do $\mathrm{C}$ é semelhante à apresentada por Arrouays \& Pelissier (1994); Salton et al. (2011) e Jobbágy \& Jackson (2000). Foi possível descrever a distribuição vertical do $\mathrm{C}$ no solo utilizando modelos matemáticos com valores de $\mathrm{R}^{2}$, na maioria das amostras, acima de 0,90, com exceção dos dados relativos aos teores de C em solos do fragmento 1 (Figura 2).

Analisando-se os desvios-padrões dos teores de $\mathrm{C}$ de cada fragmento, por camada de solo (Tabela 3), notase que, na camada de $0-10 \mathrm{~cm}$, os teores de $\mathrm{C}$ de cada fragmento se sobrepõem, o que também acontece para os fragmentos 2, 3 e 4, na camada de solo de 10-20 cm, o que não foi verificado para as demais camadas de solo. Desse modo, o uso de uma mesma regressão para a maioria dos fragmentos poderia apresentar resultados satisfatórios quanto à estimativa de $\mathrm{C}$, na camada de 0-10 cm. Porém, nas demais camadas de solo, este procedimento acarretaria erros de estimativa dos teores médios de $\mathrm{C}$ nos solos dos cinco fragmentos estudados. 
Tabela 3. Parâmetros estatísticos dos teores (\%) de C nas cinco camadas de solo, para os cinco fragmentos de cerrado sensu stricto de Januária e Bonito de Minas, MG.

\begin{tabular}{|c|c|c|c|c|c|c|}
\hline \multirow{2}{*}{ Fragmento } & \multirow{2}{*}{$\begin{array}{l}\text { Prof. de solo } \\
\text { (cm) }\end{array}$} & \multicolumn{5}{|c|}{ Parâmetro estatístico } \\
\hline & & C-médio (\%) & Desvio Padrão & CV $(\%)$ & C-Máximo (\%) & C-Mínimo (\%) \\
\hline \multirow{5}{*}{1} & $0-10$ & 0,76 & 0,27 & 35,5 & 1,23 & 0,50 \\
\hline & $10-20$ & 0,27 & 0,06 & 22,2 & 0,37 & 0,21 \\
\hline & $20-40$ & 0,22 & 0,04 & 18,2 & 0,26 & 0,18 \\
\hline & $40-60$ & 0,19 & 0,03 & 15,8 & 0,23 & 0,16 \\
\hline & $60-100$ & 0,18 & 0,02 & 11,1 & 0,22 & 0,15 \\
\hline \multirow{5}{*}{2} & $0-10$ & 0,83 & 0,22 & 26,5 & 1,04 & 0,48 \\
\hline & $10-20$ & 0,47 & 0,09 & 19,2 & 0,57 & 0,39 \\
\hline & $20-40$ & 0,40 & 0,06 & 15,0 & 0,47 & 0,32 \\
\hline & $40-60$ & 0,30 & 0,04 & 13,3 & 0,36 & 0,25 \\
\hline & $60-100$ & 0,25 & 0,04 & 16,0 & 0,30 & 0,20 \\
\hline \multirow{5}{*}{3} & $0-10$ & 0,86 & 0,36 & 41,9 & 1,38 & 0,58 \\
\hline & $10-20$ & 0,59 & 0,10 & 16,9 & 0,71 & 0,47 \\
\hline & $20-40$ & 0,50 & 0,08 & 16,0 & 0,58 & 0,41 \\
\hline & $40-60$ & 0,43 & 0,07 & 16,3 & 0,51 & 0,34 \\
\hline & $60-100$ & 0,36 & 0,02 & 5,56 & 0,39 & 0,35 \\
\hline \multirow{5}{*}{4} & $0-10$ & 0,84 & 0,15 & 17,9 & 1,07 & 0,70 \\
\hline & $10-20$ & 0,52 & 0,12 & 23,1 & 0,73 & 0,31 \\
\hline & $20-40$ & 0,41 & 0,05 & 12,2 & 0,50 & 0,35 \\
\hline & $40-60$ & 0,32 & 0,03 & 9,38 & 0,40 & 0,29 \\
\hline & $60-100$ & 0,23 & 0,02 & 8,70 & 0,27 & 0,20 \\
\hline \multirow{5}{*}{5} & $0-10$ & 1,01 & 0,07 & 6,93 & 1,13 & 0,91 \\
\hline & $10-20$ & 0,81 & 0,09 & 11,1 & 0,97 & 0,68 \\
\hline & $20-40$ & 0,70 & 0,07 & 10,0 & 0,84 & 0,63 \\
\hline & $40-60$ & 0,64 & 0,04 & 6,25 & 0,69 & 0,57 \\
\hline & $60-100$ & 0,58 & 0,02 & 3,45 & 0,60 & 0,55 \\
\hline
\end{tabular}

$\mathrm{CV}=$ coeficiente de variação.

Tabela 4. Estoque médio e desvio padrão da biomassa de serrapilheira e de raízes (em diferentes camadas de solo), para os cinco fragmentos de cerrado investigados.

\begin{tabular}{|c|c|c|c|c|c|c|c|}
\hline \multirow{3}{*}{ Fragmento } & & \multicolumn{6}{|c|}{ Biomassa (t ha $\left.{ }^{-1}\right)$} \\
\hline & & \multirow{2}{*}{ Serrapilheira } & \multicolumn{5}{|c|}{ Raiz por camada de solo } \\
\hline & & & $0-10 \mathrm{~cm}$ & $10-20 \mathrm{~cm}$ & $20-40 \mathrm{~cm}$ & $40-60 \mathrm{~cm}$ & $60-100 \mathrm{~cm}$ \\
\hline \multirow{2}{*}{1} & Média & 4,61 & 0,76 & 0,27 & 0,22 & 0,19 & 0,18 \\
\hline & $\mathrm{Dp}$ & 1,33 & 0,27 & 0,06 & 0,04 & 0,03 & 0,02 \\
\hline \multirow{2}{*}{2} & Média & 3,42 & 0,83 & 0,47 & 0,40 & 0,30 & 0,25 \\
\hline & $\mathrm{Dp}$ & 1,72 & 0,22 & 0,09 & 0,06 & 0,04 & 0,04 \\
\hline \multirow{2}{*}{3} & Média & 8,66 & 0,86 & 0,59 & 0,50 & 0,43 & 0,36 \\
\hline & $\mathrm{Dp}$ & 2,50 & 0,36 & 0,10 & 0,08 & 0,07 & 0,02 \\
\hline \multirow{2}{*}{4} & Média & 9,36 & 0,84 & 0,52 & 0,41 & 0,32 & 0,23 \\
\hline & $\mathrm{Dp}$ & 1,44 & 0,14 & 0,12 & 0,05 & 0,03 & 0,02 \\
\hline \multirow{2}{*}{5} & Média & 8,15 & 1,01 & 0,81 & 0,70 & 0,64 & 0,58 \\
\hline & $\mathrm{Dp}$ & 2,92 & 0,07 & 0,09 & 0,07 & 0,04 & 0,02 \\
\hline
\end{tabular}

$\mathrm{Dp}=$ desvio padrão. 
Tabela 5. Coeficientes de correlação linear de Pearson entre a biomassa de raiz $(0-20 \mathrm{~cm})$ e serrapilheira $\left(\mathrm{t} \mathrm{ha} \mathrm{a}^{-1}\right)$ com o teor (\%) de C na camada de solo de $0-20 \mathrm{~cm}$.

\begin{tabular}{lc}
\hline \multicolumn{1}{c}{ Biomassa } & Teor de C médio $(\mathbf{0 - 2 0} \mathbf{~ c m})$ \\
\hline Serrapilheira & $0,47^{*}$ \\
Raiz $(0-20 \mathrm{~cm})$ & $0,49^{*}$ \\
Raiz + serrapilheira & $0,51^{*}$ \\
\hline
\end{tabular}

* Significativo ao nível de $5 \%$ de probabilidade pelo teste $\mathrm{t}$.

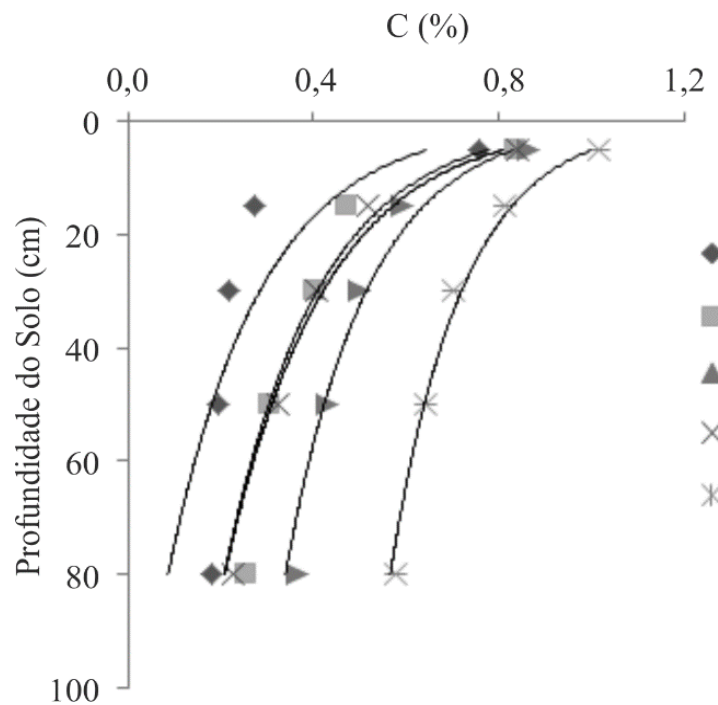

- Frag $1 \mathrm{~T}=-0,201 * \ln (\mathrm{P})+0,9675^{*}\left(\mathrm{R}^{2}=0,78^{*}\right)$

- Frag $2 \mathrm{~T}=-0,204 * * \ln (\mathrm{P})+1,1018 *\left(\mathrm{R}^{2}=0,94 * *\right)$

$\Delta$ Frag $3 \mathrm{~T}=-0,177 * * \ln (\mathrm{P})+1,1163 * * *\left(\mathrm{R}^{2}=0,97 * *\right)$

$\times$ Frag $4 \mathrm{~T}=-0,216^{* *} \ln (\mathrm{P})+1,1539 * * *\left(\mathrm{R}^{2}=0,98 * *\right)$

* Frag $5 \mathrm{~T}=-0,156 * * * \ln (\mathrm{P})+1,2496 * * *\left(\mathrm{R}^{2}=0,99 * * *\right)$

Figura 2. Distribuição vertical do teor médio de carbono em solos de cinco fragmentos de cerrado sensu stricto de Minas Gerais, para cinco camadas de solo. $*=$ significativo a $5 \% ; * *$ significativo a $1 \% ; * * *=$ significativo a $0,1 \%$ de probabilidade.

Na Figura 3 são apresentadas as médias aritméticas e ponderadas das razões (fator de concentração de $\mathrm{C}$ na superfície) entre os teores médios de $\mathrm{C}$ na camada de 0-10 e os teores médios de $\mathrm{C}$ no perfil de solo estudado $(0-100 \mathrm{~cm})$, para cada fragmento. É possível notar que, utilizando a média ponderada, essa relação varia de 1,4 a 3,8 (média de 2,13) e, quando se utiliza a média aritmética, a relação vai de 1,2 a 2,7 (média de 1,76). No entanto, como existem camadas de solos com espessura maior que $10 \mathrm{~cm}$, a média ponderada é mais indicada para essa análise. Assim, os dados mostrados na Figura 3 atestam a destacada concentração de $\mathrm{C}$ no horizonte superficial de solo, em relação às camadas mais profundas de solo, padrão já consolidado para acúmulo de C em perfis de solos em geral (Stevenson, 1994). $\mathrm{O}$ que merece destaque é o fato de alguns fragmentos apresentarem cerca de 2 vezes mais $\mathrm{C}$ em superfície do que o verificado para solos de perfis do fragmento 5 . Se de fato se materializarem os cenários de aumento da temperatura média do planeta e de intensificação de outros fatores que aceleram a decomposição da matéria orgânica do solo, os fragmentos com maior acúmulo de $\mathrm{C}$ em superfície podem se tornar mais vulneráveis em relação às perdas de $\mathrm{C}$ do solo e de seu fluxo para o ar. Considerando-se essa perspectiva, os solos do fragmento 1, num cenário de elevadas taxas de decomposição da matéria orgânica, são mais suscetíveis à decomposição da matéria orgânica do que os do fragmento 5. No fragmento 1, grande parte do C está estocado na superfície, e os teores em subsolo são relativamente baixos. No fragmento 5, os solos apresentam, para todas as camadas de solo, teores de $\mathrm{C}$ mais elevados do que os do fragmento 1. Para os solos do fragmento 5, mesmo que os teores de $\mathrm{C}$ fossem perdidos em grandes taxas na superfície, os maiores estoques de $\mathrm{C}$ em subsolo estariam mais protegidos, num cenário de efeito estufa e aquecimento global magnificados e de interferência maior desses processos sobre as taxas de decomposição da matéria orgânica presente nas camadas superficiais de solo. 


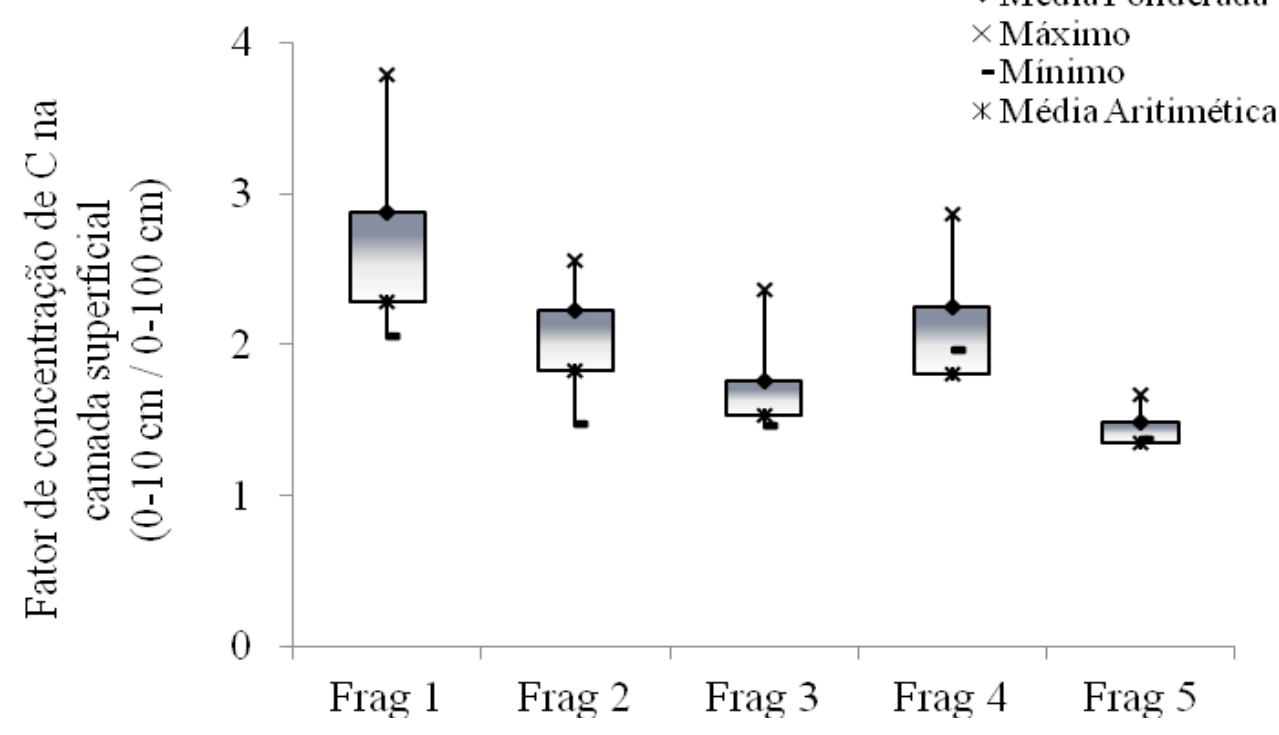

Figura 3. Fator de concentração de $\mathrm{C}$ na camada $0-10 \mathrm{~cm}$ em relação aos teores médios de $\mathrm{C}$ na camada de solo de $0-100 \mathrm{~cm}$, para os cinco fragmentos de cerrado estudados. Frag1, 2, 3, 4 e 5- fragmentos 1(Bonito de Minas), 2, 3, 4 e 5 (Januária).

O resultado das análises de correlação linear mostraram que as características climáticas (precipitação média e temperatura média) e altitude do ponto de coleta apresentaram correlação significativa com os teores de carbono, exceto para a camada de $0-10 \mathrm{~cm}$, sendo que a temperatura apresentou correlação indireta, ou negativa e as demais, direta ou positiva (Tabela 6). Estas variáveis também são citadas como correlacionantes com os teores de carbono por diversos autores, apresentando mesma tendência de correlação direta e indireta (Corazza et al., 1999; Jobbágy \& Jackson, 2000; Lal, 2005; Salton et al., 2011).

Tabela 6. Coeficientes de correlação linear de Pearson entre os teores de carbono, em diferentes camadas de solo, e as variáveis climáticas e a altitude.

\begin{tabular}{lccccc}
\hline \multirow{2}{*}{ Característica } & \multicolumn{5}{c}{ Camada de solo (cm) } \\
\cline { 2 - 6 } & $\mathbf{0 - 1 0}$ & $\mathbf{1 0 - 2 0}$ & $\mathbf{2 0 - 4 0}$ & $\mathbf{4 0 - 6 0}$ & $\mathbf{6 0 - 1 0 0}$ \\
\hline Altitude $(\mathrm{m})$ & $0,29^{\mathrm{ns}}$ & $0,57^{*}$ & $0,58^{*}$ & $0,66^{*}$ & $0,66^{*}$ \\
Precipitação $(\mathrm{mm})$ & $0,28^{\mathrm{ns}}$ & $0,64^{*}$ & $0,62^{*}$ & $0,62^{*}$ & $0,51^{*}$ \\
Temperatura $\left({ }^{\circ} \mathrm{C}\right)$ & $-0,34^{\mathrm{ns}}$ & $-0,80^{*}$ & $-0,81^{*}$ & $-0,77^{*}$ & $-0,66^{*}$ \\
\hline
\end{tabular}

ns = não significativo; $*$ significativo ao nível de $5 \%$ de probabilidade pelo teste $t$.

Os modelos apresentaram bons índices de avaliação do ajuste (Tabela 7), com exceção do modelo ajustado para a profundidade de $0-10 \mathrm{~cm}$, que apresentou baixo $\mathrm{R}^{2}$ e coeficientes não significativos, indicando que o modelo não se ajusta bem aos dados obtidos. As prováveis causas deste fato já foram discutidas anteriormente, porém, a média dos resíduos foi zero, ou seja, o modelo não apresenta tendência de subestimativa ou superestimativa e os resíduos apresentaram distribuição tendendo a normal, o que corrobora com a possibilidade de uso do mesmo para estimativas dos teores de $\mathrm{C}$ nessa camada, atentando para maiores erros nas estimativas, como pode ser observado nos gráficos de resíduos (Figura 4).

A temperatura média do ar apresentou maior correlação com os teores de $\mathrm{C}$, portanto, foi a variável de maior peso no modelo, tendência que também foi descrita e verificada por Leifeld et al. (2005), para solos da Suíça, e por Baritz et al. (2010), para solos da Europa. Segundo Sheikh et al. (2009), em regiões frias, há mais $\mathrm{C}$ estocado no solo que nas regiões mais quentes (semiáridos). Por outro lado, de acordo com Leifeld et al. (2005), há uma suspeita de que baixas temperaturas, em locais de maiores altitudes, limitem a decomposição da serrapilheira, o que, por conseguinte, pode resultar em baixo acúmulo de $\mathrm{C}$ no solo. $\mathrm{O}$ aumento da altitude também reduz a decomposição de raízes, diminuindo a entrada de $\mathrm{C}$ no solo. Vale lembrar que essas suposições se aplicam a solos em altitudes acima de $2.000 \mathrm{~m}$, portanto para regiões frias, como é o caso de solos da Suíça. 
Tabela 7. Modelos de regressão ajustados para estimativa dos teores de carbono para diferentes profundidades de solo, com seus índices de avaliação.

\begin{tabular}{|c|c|c|c|c|c|}
\hline $\begin{array}{l}\text { Profundidade } \\
\text { do solo }(\mathrm{cm})\end{array}$ & Equação matemática & $\mathbf{R}_{\text {ajust. }}^{2}$ & ME & DPEEs & RME \\
\hline $0-10$ & $\begin{array}{c}\mathrm{C}(\%)=11,1345621^{\mathrm{ns}}+\left(0,0010583^{\mathrm{ns}} \mathrm{A}\right)- \\
\left(0,083691^{\mathrm{ns}} \mathrm{T}\right)-\left(0,0081881^{\mathrm{ns} P P}\right)\end{array}$ & $10,4^{\mathrm{ns}}$ & 0 & 0,249 & 0,247 \\
\hline $10-20$ & $\begin{array}{c}\mathrm{C}(\%)=22,9957350^{* * *}+\left(0,0019401^{* * *} \mathrm{~A}\right)- \\
\left(0,1905508^{* * *} \mathrm{~T}\right)-\left(0,0178862^{* * *} \mathrm{PP}\right)\end{array}$ & $72,6^{* * *}$ & 0 & 0,109 & 0,108 \\
\hline $20-40$ & $\begin{array}{c}\mathrm{C}(\%)=22,5099488^{* * *}+\left(0,0018459^{* * *} \mathrm{~A}\right)- \\
\left(0,1788626^{* * *} \mathrm{~T}\right)-\left(0,0173714^{* * *} \mathrm{PP}\right)\end{array}$ & $77,7^{* * * *}$ & 0 & 0,085 & 0,085 \\
\hline $40-60$ & $\begin{array}{c}C(\%)=21,2944333^{* * *}+\left(0,0019969^{* * *} A\right)- \\
\left(0,159577^{* *} T\right)-\left(0,0168239^{* * *} P P\right)\end{array}$ & $82,0^{* * * *}$ & 0 & 0,070 & 0,070 \\
\hline $60-100$ & $\begin{array}{c}\mathrm{C}(\%)=25,8723003^{* * *}+\left(0,0024555^{* * *} \mathrm{~A}\right)- \\
\left(0,162788^{* * *} \mathrm{~T}\right)-\left(0,0212794^{* * *} \mathrm{PP}\right)\end{array}$ & $87,4^{* * * *}$ & 0 & 0,055 & 0,055 \\
\hline
\end{tabular}

ns = não significativo; $* * *$ significativo ao nível de $0,1 \%$ de probabilidade. $\mathrm{A}=$ altitude de cada ponto de coleta $(\mathrm{m}) ; \mathrm{T}=$ temperatura média do ar $\left({ }^{\circ} \mathrm{C}\right)$; $\mathrm{PP}=$ precipitação média $(\mathrm{mm}) ; \mathrm{ME}=$ média dos resíduos; $\mathrm{RME}=$ raiz do erro médio da estimativa; DPEEs = desvio padrão da estimativa.
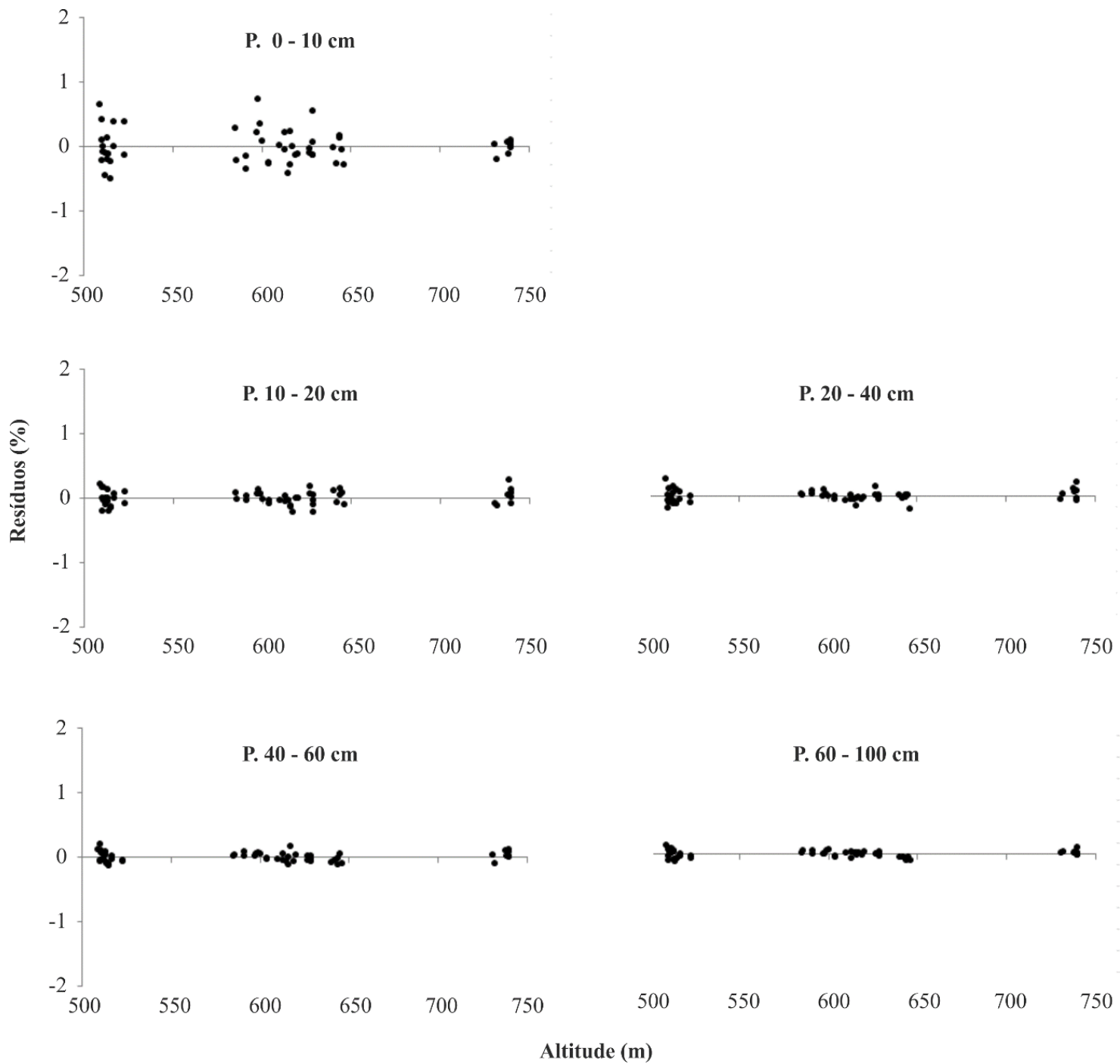

Figura 4. Distribuição dos resíduos dos modelos matemáticos para os teores de carbono em função da camada de solo (P. = profundidade do solo). 
Embora as amplitudes dos dados de precipitação, temperatura e altitude utilizadas no estudo de modelagem do $\mathrm{C}$ em solo sejam relativamente estreitas, as mesmas compreendem o intervalo encontrado em uma área de aproximadamente 3,11 milhões de hectares, denominada por Scolforo \& Carvalho (2008) como sub-bacia 9 do Rio São Francisco (SF 9). Segundo esses autores, próximo a 1 milhão de hectares desta área foi classificada como cerrado sensu stricto, de modo que, por tudo isso, as equações matemáticas disponibilizadas para estimativa do $\mathrm{C}$ em solo assumem importância, dado que elas permitem inventariar de modo mais eficiente $\mathrm{e}$ preciso o $\mathrm{C}$ armazenado em áreas de cerrado mineiro.

\section{Conclusões}

Os teores de C, em função da profundidade do solo, ajustaram-se a equações logarítmicas, sendo que os modelos aqui disponibilizados são específicos para cada fragmento de cerrado.

A razão dos teores de C-solo na camada de $0-10 \mathrm{~cm}$ com o C médio no perfil $(0-100 \mathrm{~cm})$ varia na faixa de 1,3 a 3,8, demonstrando, entre os cinco fragmentos de cerrado, grande variação nos teores de $\mathrm{C}$ armazenado na superfície em relação ao C-subsolo.

Isoladamente, a temperatura, a altitude e a precipitação pouco explicam as variações dos teores de $\mathrm{C}$ nas diferentes camadas de solo, mas, quando associados, esses fatores explicam, à exceção da camada de $0-10 \mathrm{~cm}$, mais de $72 \%$ das variações do $\mathrm{C}$ em perfis de solos dos fragmentos de cerrado.

Para estimar os teores de $\mathrm{C}$ nos fragmentos de cerrado é preciso, portanto, considerar a camada de solo amostrada, as unidades amostrais de coleta de solo, as variáveis climáticas e a altitude dos locais de coleta de solo.

\section{Agradecimentos}

Os autores agradecem aos coordenadores do Inventário Florestal de Minas Gerais, pela disponibilização dos dados utilizados neste trabalho. Ao Conselho Nacional de Desenvolvimento Científico e Tecnológico - CNPq (Processo 308592/2011-5), Fundação de Amparo à Pesquisa do Estado de Minas Gerais - FAPEMIG (Processo CAG - APQ-00291-11) e à Coordenação de Aperfeiçoamento de Pessoal de Nível Superior -
CAPES, pela concessão de bolsa de Doutorado ao $1^{\circ}$ autor. Ao Ewerton, pela finalização das figuras. Especial agradecimento a todo equipe de alunos e estagiários do $\mathrm{DCF} / \mathrm{LEMAF}$ e demais profissionais que, em campo e no laboratório, contribuíram para a coleta de amostras e geração dos dados utilizados neste estudo.

\section{Referências}

ADUAN, E. A.; VILELA, M. F.; KLINK, C. A. Ciclagem de carbono em ecossistemas terrestres: o caso do cerrado brasileiro. Planaltina: Embrapa Cerrados, 2003. 30 p.

ARROUAYS, D.; PELISSIER, P. Modeling carbon storage profiles in temperate forest humic loamy soils of France. Soil Science, Baltimore, v. 157, n. 157, p. 185-192, 1994.

BAYER, C.; MARTIN-NETO, L.; MIELNICZUK, J.; PAVINATO,A.; DIECKOW, J. Carbon sequestration in two Brazilian Cerrado soils under no-till. Soil \& Tilage Research, Amsterdam, n. 86, p. 237-245, 2006.

BARITZ, R.; SEUFERT, G.; MONTANARELLA, L.; RANST, E.V. Carbon concentrations and stocks in forest soils of Europe. Forest Ecology and Management, Amsterdam, p. 262-277, 2010.

BENITES, V. M.; MACHADO, P.; FIDALGO, E. C. C.; COELHO, M. R.; MADARI, B. E. Pedotransfer functions for estimating soil bulk density from existing soil survey reports in Brazil. Geoderma, Amsterdam, n. 139, p. 90-97, 2007.

CARVALHO, L. G.; OLIVEIRA, M. S. de; ALVES, M. de C.; VIANELLO, R. L.; SEDIYAMA, G. C.; NETO, P. C. DANTAS, A. A. A. Clima. In: SCOLFORO, J. R.; CARVALHO, L. M. T.; OLIVEIRA, A. D. Zoneamento ecológico-econômico do estado de Minas Gerais: componentes geofísico e biótico. Lavras: UFLA, 2008. p. 89-101.

CHARNET, R.; FREIRE, C.A. de; CHARNET, E. M. R.; BONVINO, $\mathrm{H}$. Análise de modelos de regressão linear: com aplicações. 2. ed. Campinas: Ed da Unicamp, 2008. 356 p.

CORAZZA, E. J.; SILVA, J. E.; RESCK, D. V. S.; GOMES, A. C. Comportamento de diferentes sistemas de manejo como fonte ou depósito de carbono em relação à vegetação de cerrado. Revista Brasileira de Ciência do Solo, Campinas, n. 23, p. 425-432, 1999.

CURI, N.; MARQUES, J. J. G.; MARQUES, A. F. S. M.; FERNANDES, E. I. Solos, geologia, relevo e mineração. In: SCOLFORO, J. R.; CARVALHO, L. M. T.; OLIVEIRA, A. D. Zoneamento ecológico-econômico do estado de Minas Gerais: componentes geofísico e biótico. Lavras: UFLA, 2008. p. 73-88.

DICK, W. A.; GREGORICH, E. G. Developing and maintaining soil organic matter levels. In: SCHJONNING, P.; ELMHOLT, S.; CHRISTENSEN, B. T. Managing soil quality: challenges in modern agriculture. Wallingford: CABI Publishing, 2004. p. 103-120.

FREIXO, A. A.; MACHADO, P. L. O. A.; GUIMARÃES, C. M.; SILVA, C. A.; FADIGAS, F. S. Estoque de carbono e nitrogênio e distribuição de frações orgânicas de latossolo do cerrado sob diferentes sistemas de cultivo. Revista Brasileira de Ciência do Solo, Campinas, n. 26, p. 424-434, 2002. 
FUNDAÇÃO DE CIÊNCIA, APLICAÇÕES E TECNOLOGIAS ESPACIAIS. Segundo inventário brasileiro de emissões e remoções antrópicas de gases de efeito estufa: relatórios de referência: emissões de gases de efeito estufa no setor uso da terra, mudança do uso da terra e florestas. Brasília, DF: Ministério da Ciência e Tecnologia, 2010. Disponível em: <http://www.mct.gov. br/upd_blob/0215/215990.pdf>. Acesso em: 20 dez. 2012.

JOBBÁGY, E. G.; JACKSON, R. B. The vertical distribution of soil organic carbon and its relation to climate and vegetation. Ecological Applications, Ithaca, n. 10, p. 423-436, 2000.

KAUR R.; KUMAR, S.; GURUNG, H.P. Pedotransfer functions (PTFs) for estimating soil bulk density from basic soil data and its comparison with existing PTFs. Australian Journal of Soil Research, Melbourne, 40, p. 847-857, 2002.

KAY, B.D.; SILVA, A. P. da.; BALDOCK J. A. Sensitivity of soil structure to changes in organic carbon content: Predictions using pedotransfer functions. Canadian Journal Soil Science, Ottawa, n. 77, p. 655-667, 1997.

KNOPS, J. M. H.; BRADLEY, K. L. Soil carbon and nitrogen accumulation and vertical distribution across a 74-year chronosequence. Soil Science Society America Journal, Madison, v. 73, p. 2096-2104, 2009.

LEIFELD, J.; BASSIN, J.; FUHRER, J. Carbon stocks in Swiss agricultural soils predicted by land-use, soil characteristics, and altitude. Agriculture, Ecosystems \& Environment, Amsterdam, v. 105, p. 255-266, 2005.

LAL, R. he potential of soils of the tropics to sequester carbon and mitigate the greenhouse effect. Advances in Agronomy, Amsterdam, n. 76, p. 1-30, 2002.

LAL, R. Forest soils and carbon sequestration. Forest Ecology and Management, Amsterdam, v. 220, p. 242-258, 2005.

LAL, R. Carbon sequestration. Philosophical Transactions of the Royal Society B, London, v. 363, p. 815-830, 2008.

MARCOLIN, C. D.; KLEIN, V. A. Determinação da densidade relativa do solo por uma função de pedotransferência para a densidade do solo máxima. Acta Scientiarum: Agronomy, Maringá, v. 33, p. 349-354, 2011.

MARIMON JÚNIOR, B. H.; HARIDASAN, M. Comparação da vegetação arbórea e características edáficas de um cerradão e um cerrado Sensu Stricto em áreas adjacentes sobre solo distrófico no leste de Mato Grosso, Brasil. Acta Botanica Brasilica, Porto Alegre, v. 19, p. 913-926, 2005.

MARTIN, M. P.; SEEN, D. L.; BOULONNE, L.; JOLIVET, C.; NAIR, K. M.; BOURGEON, G.; ARROUAYS, D. Optimizing pedrotransfer functions for estimating soil bulk density using boosted regression trees. Soil Science Society of America, New York, v. 73, p. 485-493, 2009.

McBRATNEY, A. B.; MINASNY, B.; TRANTER, G. Necessary meta-data for pedotransfer functions. Geoderma, Amsterdam, v. 160, p. 627-629, 2010.

MORAIS, V. A.; SCOLFORO, J. R. S.; SILVA, C. A.; MELLO, J. M.; GOMIDE, L. R.; OLIVEIRA, A. D. Carbon and biomass stocks in a fragment of cerradão in Minas Gerais state, Brazil. Cerne, Lavras, v. 19, n. 2 , p. $237-245,2013$.

Pesq. flor. bras., Colombo, v. 33, n. 76, p. 343-354, out./dez. 2013
OLIVEIRA, J. R. A.; MENDES, I C. C.; VIVALDI, L. Carbono da biomassa microbiana em solos de cerrado sob vegetação nativa e sob cultivo: avaliação dos métodos fumigação-incubação e fumigação-extração. Revista Brasileira de Ciência do Solo, Campinas, v. 25, p. 863-871, 2001.

PAIVA, A. O.; FARIA, G. E. Estoque de carbono do solo sob Cerrado Sensu Stricto no Distrito Federal, Brasil. Revista Tropica: Ciências Agrárias e Biológicas, Chapadinha, v. 1, p. 59-65, 2007.

PAIVA, A. O.; REZENDE, A. V.; PEREIRA, R. S. Estoque de carbono em cerrado sensu stricto do Distrito Federal. Revista Árvore, Viçosa, MG, v. 35, p. 527-538, 2011.

QUIDEAU, S. A. Organic matter accumulation. Encyclopedia of Soil Science, v. 26, p. 1-4, 2002.

R DEVELOPMENT CORE TEAM. R: A language and environment for statistical computing.Vienna: R Foundation for Statistical Computing, 2008. Disponível em: <http://www.R-project.org>. Acesso em: 22 out. 2011.

RIBEIRO, J. F.; WALTER, B. M. T. Fitofisionomias do bioma Cerrado. In: SANO, S. M.; ALMEIDA, S. P.; RIBEIRO, J. F. (Ed.). Cerrado: ecologia e flora. Planaltina: Embrapa Cerrados, 2008. v. 1. p.89-168.

RICHTER, D. D.; MARKEWITZ, D.; TRUMBORE, S. E.; WELLS, C. G. Rapid accumulation and turnover of soil carbon in a reestablishing forest. Nature, London, v. 400, p. 56-58, 1999.

ROSCOE, R.; MACHADO, P. L. O. de A. Fracionamento físico do solo em estudos da matéria orgânica. Dourados: Embrapa Agropecuária Oeste; Rio de Janeiro: Embrapa Solos, 2002. 86 p.

SALTON, J. C.; MIELNICZUK, J.; BAYER, C.; FABRICIO, A. C.; MACEDO, M. C. M.; BROCH, D. L. Teor e dinâmica do carbono no solo em sistemas de integração lavoura-pecuária. Pesquisa Agropecuária Brasileira, Brasília, DF, v. 46, p. 1349-1356, 2011.

SCOLFORO, J. R.; MELLO, J. M. de; OLIVEIRA, A. D. de; CARVALHO, L. M. T de; ACERBI JUNIOR, F. W. Amostragem e caracterização dos fragmentos inventariados. In: SCOLFORO, J. R. S.; MELLO, J. M. de; OLIVEIRA, A. D. de. Inventário florestal de Minas Gerais: florística, estrutura, diversidade, similaridade, distribuição diamétrica e de altura, volumetria, tendências de crescimento e áreas aptas para manejo florestal. Lavras: UFLA, 2008. p. 1-78.

SCOLFORO, J. R.; CARVALHO, L. M. T. Mapeamento e inventário da flora e dos reflorestamentos de Minas Gerais. Lavras: Ed. da UFLA, 2008. 287 p.

SHEIKH, M.; KUMAR, M.; BUSSMANN, R. W. Altitudinal variation in soil organic carbon stock in coniferous subtropical and broadleaf temperate forests in Garhwal Himalaya. Carbon balance and management, United Kingdom, v. 4, n. 6, p. 1-6, 2009.

STEVENSON, F. J. Humus chemistry: genesis, composition, reactions. New York: John Wiley \& Sons, 1994. 496 p.

TOMASELLA, J.; HODNETT, M. Pedotransfer functions for tropical soils. Developments in Soil Science, Amsterdam, v. 30, p. 415- 429, 2004.

ZINN, Y. L.; LAL, R.; RESCK, D. V. S. Texture and organic carbon relations described by a profile pedotransfer function for Brazilian Cerrado soils. Geoderma, Amsterdam, v. 127, p. 168-173, 2005. ZHOU, G.; LIU, S.; LI, Z.; ZHANG, D.; TANG, X.; ZHOU, C.; YAN, J.; MO, J. Old-growth forest can accumulate carbon in soils. Science, New York, v. 314, p. 1417-1418, 2006. 